\title{
CONVERSÃO E BALANÇO ENERGÉTICO DE SISTEMAS DE ROTAÇÃO DE CULTURAS PARA TRITICALE, SOB PLANTIO DIRETO
}

\author{
ENERGY AND BALANCE CONVERSION OF CROP ROTATION \\ SYSTEMS FOR TRITICALE, UNDER NO-TILLAGE
}

\author{
Henrique Pereira dos Santos ${ }^{1}$ João Carlos Ignaczak ${ }^{2}$ \\ Julio Cesar Barreneche Lhamby ${ }^{3}$ Augusto Carlos Baier ${ }^{3}$
}

RESUMO

Na agricultura moderna, interessam sistemas de produção eficientes no uso da energia. Objetivou-se avaliar a conversão e o balanço energético de cinco sistemas de rotação de culturas envolvendo o triticale. Os sistemas avaliados, no período de 1987 a 1991, foram: I (triticale/soja), II (triticale/soja e aveia preta/soja), III (triticale/soja e ervilhaca/milho), IV (triticale/soja, ervilhaca/milho e aveia preta/soja) e V (triticale/soja, triticale/soja, ervilhaca/milho e aveia preta/soja). Em 1990, nos sistemas II, IV e V, a aveia preta foi substituída por aveia branca. $O$ experimento foi estabelecido em plantio direto, em delineamento de blocos ao acaso, com três repetiç̃es e parcelas com área útil de $24 \mathrm{~m}^{2}$. Na média do período de 1987 a 1989, o sistema III apresentou conversão (9,30) e balanço energético $(23.860$ Mcal/ha) maiores do que os demais sistemas estudados (I: 5,38, II: 5,02, IV: 8,12 e V: 7,37; I: 18.067 Mcal/ha, II: 13.790 Mcal/ha, IV: $19.875 \mathrm{Mcal} / \mathrm{ha}$ e $\mathrm{V}: 19.264 \mathrm{Mcal} / \mathrm{ha}$, respectivamente). Nesse período, as condições climáticas transcorreram normalmente. Na média do período de 1990 a 1991, não houve diferenças significativas entre as médias para conversão e para balanço energético. Nesse período, as condições climáticas foram adversas às culturas em estudo.

Palavras-chave: rendimento de grãos, aveia branca, aveia preta ervilhaca, milho, soja.

\section{SUMMARY}

Efficient energy conversion production systems are important for modern agriculture. The objetive was to evaluate the energy conversion and balance of five rotation systems that included triticale. The evaluated systems, from 1987 to 1991, were: I (triticale/soybean), II (triticale/soybean and black oats/soybean), III (triticale/soybean and common vetch/corn), IV (triticale/soybean, common vetch/corn, and black oats/soybean), and $V$ (triticale/soybean, triticale/soybean, common vetch/corn, and black oats/ soybean). In 1990, black oats was replaced by white oats in systems $I I, I V$, and V. The experiment was set up under no-tillage, using a randomized block design with three replications and plots totalizing $24 \mathrm{~m}^{2}$. On average, for the period 1987 to 1989, system III showed higher energy conversou (9.30) and balance (23.860 Mcal/ha), as compared to the othen studied systems (I: 5.38, II: 5.02, IV: 8.12 e V: 7.37; I: 18.067 Mcal/ha, II: $13.790 \mathrm{Mcal} / \mathrm{ha}, \mathrm{IV}: 19.875 \mathrm{Mcal} / \mathrm{ha}$ e V: 19.264 Mcal/ha, respectivily). In this period, the climatic conditions were normal. On average, the period 1990 to 1991, there were no significant differences between energy conversion and balance means. In this period, climatic conditions were adverse.

Key words: yield, white oast, black oats, common vetch, corn, soybean.

\section{INTRODUÇÃO}

No último século, o uso da energia tem se intensificado a tal ponto que se começa a sentir as grandes limitações econômicas com a sua escassez (IGUE, 1980). O aumento do uso da energia consumida na agricultura, combinado com a elevação dos custos e com possíveis armazenamentos para o futuro têm levado à necessidade de se obter sistemas agropecuários mais eficientes na utilização desse recurso não renovável (exemplo: combustíveis, fertilizantes, fungicidas, herbicidas e inseticidas) (ZENTNER et al., 1984).

A produção altamente tecnificada de cereais e de oleaginosas, para consumo humano, prin-

\footnotetext{
${ }^{1}$ Engenheiro Agrônomo, Doutor, Bolsista do CNPq, Embrapa-Centro Nacional de Pesquisa de Trigo, CP 451, 990O1-970, Passo Fundo, RS. E-mail: hpsantos@ cnpt.embrapa.br. Autor para correspondência.

${ }^{2}$ Engenheiro Agrônomo, Mestre, Embrapa-CNPT.

${ }^{3}$ Engenheiro Agrônomo, Doutor, Embrapa-CNPT. 
cipalmente nas regiões temperadas, como nos Estados Unidos da América, no Canadá, na Alemanha, na França, na Inglaterra e no Brasil, exige um elevado consumo de energia não renovável (WHITE, 1975). Na região tropical, a obtenção desses mesmos produtos ocorre com menores dispêndios de energia, devido a condições de alta luminosidade e de baixo uso de insumos, o que os torna energeticamente mais favoráveis (WILSON \& BRIGSTOCKE, 1980). Um sistema tecnologicamente adequado, que busca maior eficiência na utilização da energia não renovável e no lucro, somente poderá ser estabelecido mediante uma racionalização das atividades executadas na propriedade (IGUE, 1980).

As chamadas tecnologias modernas, normalmente, incluem práticas (utilização de combustíveis, de corretivos, de fertilizantes, de fungicidas, de herbicidas e de inseticidas) que são recomendadas aos produtores, a fim de aumentar a produtividade e otimizar a relação custo-benefício (IGUE, 1980). Nesse sentido, têm sido desenvolvidos trabalhos, em vários países, para avaliar a eficiência da conversão energética em grãos entre espécies ou em produtos de origem animal (carne ou leite) (BERARDI, 1978; PIMENTEL, 1980; WILSON \& BRIGSTOCKE, 1980; QUESADA et al., 1987; BOHRA et al., 1990). Através desses trabalhos, verifica-se que as fontes de energia utilizadas variam de um país para outro, segundo seus costumes, recursos disponíveis e grau de desenvolvimento.

Há poucas pesquisas relativas a estudos com conversão energética e com balanço energético em sistemas de rotação de culturas. ZENTNER $\boldsymbol{e t}$ al. (1984, 1989), no Estado de Saskatchewan, no Canadá, ao pesquisarem a conversão energética e o balanço energético, em 12 e em 18 anos de cultivo, respectivamente, encontraram, somente no primeiro período estudado, diferenças significativas para conversão energética entre os sistemas de rotação de culturas para trigo.

No Estado do Rio Grande do Sul, SANTOS et al. (1995), pesquisando índices de produtividade cultural, com dez anos de cultivo, sob preparo convencional de solo no inverno e sob plantio direto no verão, não encontraram diferenças significativas entre os sistemas de rotação de culturas para trigo. SANTOS et al. (1996a, 1996b), no Estado do Paraná, durante dez anos, sob plantio direto, observaram índices de produtividade cultural mais elevados para os sistemas com rotação de culturas, em relação à monocultura de cevada e de trigo.

O objetivo deste trabalho foi avaliar a conversão energética e o balanço energético de sistemas de rotação de culturas para triticale, em Passo Fundo, RS.

\section{MATERIAL E MÉTODOS}

No presente trabalho, foram utilizados os dados obtidos no experimento de sistemas de rotação de culturas para triticale, instalado na EmbrapaCentro Nacional de Pesquisa de Trigo (CNPT), município de Passo Fundo, RS, de 1987 a 1991, em latossolo vermelho escuro distrófico (BRASIL, 1973).

Cinco sistemas de produção, tendo o triticale como cultura principal, foram avaliados no experimento: sistema I (triticale/soja), sistema II (triticale/soja e aveia preta/soja), sistema III (triticale/soja e ervilhaca/milho), sistema IV (triticale/soja, ervilhaca/milho e aveia preta/soja) e sistema V (triticale/soja, triticale/soja, ervilhaca/milho e aveia preta/soja). Em 1990, nos sistemas II, IV e V, a aveia preta foi substituída por aveia branca (tabela 1). Para permitir a avaliação do efeito do ano, o experimento teve uma combinação de tratamento em que todas as espécies em estudo estão presentes a cada ano, permitindo que o efeito dos sistemas de rotação seja separado do efeito que as condições climáticas de cada ano causam no desempenho das culturas.

As parcelas individuais, compostas pelas culturas, de inverno ou de verão, componentes dos sistemas, tinham a área útil de $24 \mathrm{~m}^{2}$ ( $3 \mathrm{~m}$ de largura

Tabela 1 - Sistemas de rotação de culturas para triticale, com espécies de inverno e de verão, em plantio direto. Embrapa-CNPT, Passo Fundo

\begin{tabular}{|c|c|c|c|c|c|}
\hline \multirow{2}{*}{$\begin{array}{l}\text { Sistema de } \\
\text { rotação }\end{array}$} & \multicolumn{5}{|c|}{ Ano } \\
\hline & 1987 & 1988 & 1989 & 1990 & 1991 \\
\hline Sistema I & $\mathrm{Tcl} / \mathrm{S}$ & $\mathrm{Tcl} / \mathrm{S}$ & $\mathrm{Tcl} / \mathrm{S}$ & $\mathrm{Tcl} / \mathrm{S}$ & $\mathrm{Tcl} / \mathrm{S}$ \\
\hline \multirow[t]{2}{*}{ Sistema II } & $\mathrm{Tcl} / \mathrm{S}$ & $\mathrm{Ap} / \mathrm{S}$ & $\mathrm{Tcl} / \mathrm{S}$ & $\mathrm{Ab} / \mathrm{S}$ & $\mathrm{Tcl} / \mathrm{S}$ \\
\hline & $\mathrm{Ap} / \mathrm{S}$ & $\mathrm{Tcl} / \mathrm{S}$ & $\mathrm{Ap} / \mathrm{S}$ & $\mathrm{Tcl} / \mathrm{S}$ & $\mathrm{Ab} / \mathrm{S}$ \\
\hline \multirow[t]{2}{*}{ Sistema III } & $\mathrm{Tcl} / \mathrm{S}$ & $\mathrm{E} / \mathrm{M}$ & $\mathrm{Tcl} / \mathrm{S}$ & $\mathrm{E} / \mathrm{M}$ & $\mathrm{Tcl} / \mathrm{S}$ \\
\hline & $\mathrm{E} / \mathrm{M}$ & $\mathrm{Tcl} / \mathrm{S}$ & $\mathrm{E} / \mathrm{M}$ & $\mathrm{Tcl} / \mathrm{S}$ & $\mathrm{E} / \mathrm{M}$ \\
\hline \multirow[t]{3}{*}{ Sistema IV } & $\mathrm{Tcl} / \mathrm{S}$ & $\mathrm{Ap} / \mathrm{S}$ & $\mathrm{E} / \mathrm{M}$ & $\mathrm{Tcl} / \mathrm{S}$ & $\mathrm{E} / \mathrm{M}$ \\
\hline & $\mathrm{Ap} / \mathrm{S}$ & $\mathrm{E} / \mathrm{M}$ & $\mathrm{Tcl} / \mathrm{S}$ & $\mathrm{E} / \mathrm{M}$ & $\mathrm{Ab} / \mathrm{S}$ \\
\hline & $\mathrm{E} / \mathrm{M}$ & $\mathrm{Tcl} / \mathrm{S}$ & $\mathrm{Ap} / \mathrm{S}$ & $\mathrm{Ab} / \mathrm{S}$ & $\mathrm{Tcl} / \mathrm{S}$ \\
\hline \multirow[t]{4}{*}{ Sistema V } & $\mathrm{Tcl} / \mathrm{S}$ & $\mathrm{Tcl} / \mathrm{S}$ & Ap/S & $\mathrm{Ab} / \mathrm{S}$ & $\mathrm{Tcl} / \mathrm{S}$ \\
\hline & $\mathrm{Tcl} / \mathrm{S}$ & $\mathrm{Ap} / \mathrm{S}$ & $\mathrm{E} / \mathrm{M}$ & $\mathrm{Tcl} / \mathrm{S}$ & $\mathrm{Tcl} / \mathrm{S}$ \\
\hline & $\mathrm{Ap} / \mathrm{S}$ & $\mathrm{E} / \mathrm{M}$ & $\mathrm{Tcl} / \mathrm{S}$ & $\mathrm{Tcl} / \mathrm{S}$ & $\mathrm{E} / \mathrm{M}$ \\
\hline & $\mathrm{E} / \mathrm{M}$ & $\mathrm{Tcl} / \mathrm{S}$ & $\mathrm{Tcl} / \mathrm{S}$ & $\mathrm{E} / \mathrm{M}$ & $\mathrm{Ab} / \mathrm{S}$ \\
\hline
\end{tabular}

$\mathrm{Ab}=$ aveia branca (UFRGS 7); Ap = aveia preta (Comum); E = ervilhaca (Comum); $\mathrm{M}=$ milho (AG 64 A e XL 560); $\mathrm{S}=$ soja (BR 4); Tcl = triticale (BR 1 e BR 4). 
x $8 \mathrm{~m}$ de comprimento). $\mathrm{O}$ delineamento experimental foi o de blocos ao acaso, com três repetições.

Antes da instalação do experimento (abril de 1986), a área foi descompactada (com subsolador de hastes), e 4,0 $\mathrm{t} \mathrm{ha}^{-1}$ de calcário foram aplicadas para corrigir a acidez de solo $(5,5)$. Inicialmente, a aveia preta foi semeada (1986) e ceifada com rolo faca na plena floração. A seguir, a soja foi semeada (1986) em plantio direto. As culturas de inverno e as de verão foram estabelecidas com semeadora comercial, em plantio direto. Em maio de 1989, antes da semeadura de inverno, foram aplicados na superfície, sem incorporar, 2,8 $\mathrm{ha}^{-1}$ de calcário com PRNT $70 \%$, correspondente à dose máxima recomendada para solo sob plantio direto.

A conversão energética dos sistemas estudados resulta da divisão da energia produzida pela consumida, em cada sistema. $\mathrm{O}$ balanço energético dos sistemas estudados resulta da diferença entre a energia produzida e a consumida, em cada sistema. Como energia produzida considerou-se a transformação do rendimento de grãos em energia. Como energia consumida considerou-se a soma dos coeficientes energéticos equivalentes aos corretivos, fertilizantes, sementes, fungicidas, herbicidas e inseticidas, utilizados em cada sistema, bem como a energia consumida pelas operações de semeadura, adubação, aplicação de produtos, adubação nitrogenada e colheita. No caso da aveia preta (1987 a 1989) e da ervilhaca (1987 a 1991), foi considerada como rendimento a contribuição ao solo de $90 \mathrm{~kg} / \mathrm{ha}$ de $\mathrm{N}$ (DERPSCH $\boldsymbol{e}$ t al., 1985, DERPSCH \& CALEGARI, 1992). Para as culturas produtoras de grãos, não foi considerada a possível contribuição de $\mathrm{N}$ ao solo. Como os valores da conversão energética ou do balanço energético foram relativamente baixos, os dados foram transformados em Mcal (Megaquicalorias: kcal x 1.000).

Para o cálculo de diversos índices envolvendo sistemas, rendimento de grãos e operações de campo, foram utilizados dados e orientações gerados por HEICHEL, 1980, PIMENTEL, 1980, MARCHIORO, 1985, MELLO, 1986 e EMBRAPA, 1991.

Foram efetuadas as análises de variância da conversão e de balanço energético dentro de cada ano (inverno + verão) e na média conjunta dos anos nos dois períodos, 1987 a 1989 e 1990 a 1991. A análise de variância conjunta foi aplicada a esses dois períodos, devido às alterações efetuadas nos sistemas II, IV e V a partir de 1990. Na análise de variância anual, consideraram-se como tratamentos as culturas individuais componentes dos sistemas em estudo. Nas análises conjuntas, considerou-se o efeito tratamento como fixo, e o efeito do ano, como aleatório. A avaliação dos sistemas de rotação, em todas as análises, foi realizada através do teste $\mathrm{F}$, usando-se contrastes que incluem os diferentes tratamentos dos sistemas de rotação envolvidos em cada comparação. Essa metodologia de contrastes (STEEL \& TORRIE, 1980) compara os sistemas dois a dois em uma unidade de base homogênea.

\section{RESULTADOS E DISCUSSÃO}

As médias da conversão energética e do balanço energético, anuais e dos períodos (1987 a 1989 e 1990 a 1991), e as comparações estatísticas através de contrastes, dos cinco sistemas de produção para triticale, podem ser observadas nas tabelas 2 e 3 . As análises de variância conjunta para conversão energética e para balanço energético, nos dois períodos (1987 a 1989 e 1990 a 1991), apresentaram diferenças significativas para os efeitos anos e para a interação anos x tratamentos. Ocorreram diferenças significativas somente entre as médias dos sistemas para conversão energética e para balanço energético no primeiro período.

Levando-se em conta a comparação da conversão energética anual (inverno + verão) do primeiro período (1987 a 1989), ocorreram diferenças significativas nos três anos estudados (tabela 2). Nesse período, os sistemas de rotação de culturas (III, IV e V) que continham o milho como um dos seus componentes foram mais eficientes na conversão de energia do que a monocultura (triticale/soja). $\mathrm{O}$ baixo desempenho energético de uma das culturas (ervilhaca) de cobertura de solo, no inverno foi compensado, em parte, principalmente, pelo maior desempenho do milho, no verão. Além disso, a cultura de milho produziu rendimento de grãos relativamente elevado, variando de $6.249 \mathrm{~kg} / \mathrm{ha}$ a $8.312 \mathrm{~kg} / \mathrm{ha}$. Por outro lado, a soja produziu rendimento relativamente elevado somente no ano de 1988 (de $2.776 \mathrm{~kg} / \mathrm{ha}$ a $3.493 \mathrm{~kg} / \mathrm{ha}$ ). Nos anos de 1987 e 1989, o rendimento de grãos dessa leguminosa foi baixo (de $1.033 \mathrm{~kg} / \mathrm{ha}$ a $2.312 \mathrm{~kg} / \mathrm{ha}$ ). Isso, por si só, explica o baixo desempenho energético dos sistemas I e II. A cultura de triticale, que fez parte de todos os sistemas, igualmente produziu relativamente elevado $(2.013 \mathrm{~kg} / \mathrm{ha}$ a $4.513 \mathrm{~kg} / \mathrm{ha})$.

Em função do observado acima, as médias comparadas duas a duas, de 1987 a 1989, mostram que os sistemas III $(9,30)$, IV $(8,12)$ e V $(7,37)$ foram superiores aos sistemas I $(5,38)$ e II $(5,02)$, para conversão energética (tabela 2 ). Por sua vez, o sistema I não diferiu do sistema II. O sistema III foi superior aos sistemas IV e V, e o sistema IV foi superior ao sistema V. Nesse período, o sistema III foi o que apresentou maior conversão energética 
Tabela 2 - Conversão energética e sua comparação em cinco sistemas de rotação de culturas para triticale, no ano (inverno + verão) e na média conjunta pelo teste $\mathrm{F}$, utilizando-se o método de contrastes.

\begin{tabular}{|c|c|c|c|c|c|c|c|c|c|c|c|c|c|c|c|}
\hline \multirow[t]{2}{*}{ Ano } & \multicolumn{15}{|c|}{ Sistema de rotação ${ }^{1}$} \\
\hline & $\mathrm{I}$ & II & III & IV & $\mathrm{V}$ & I x II & I x III & I x IV & $\mathrm{I} \times \mathrm{V}$ & II x III & II x IV & II $x \mathrm{~V}$ & III x IV & III $x \mathrm{~V}$ & IV $x \mathrm{~V}$ \\
\hline & & & & & & & & & \multicolumn{7}{|c|}{ Contrastes entre sistemas } \\
\hline 1987 & 5,08 & 4,28 & 8,07 & 7,40 & 6,36 & ns & $* *$ & $* *$ & $*$ & $* *$ & $* *$ & $* *$ & ns & $* *$ & $*$ \\
\hline 1988 & 5,59 & 6,14 & 9,91 & 9,06 & 8,36 & ns & $* *$ & $* *$ & $* *$ & $* *$ & $* *$ & $* *$ & ns & $*$ & ns \\
\hline 1989 & 5,47 & 4,63 & 9,91 & 7,89 & 7,38 & ns & $* *$ & ** & $* *$ & $* *$ & $* *$ & $* *$ & $* *$ & $* *$ & ns \\
\hline Média & 5,38 & 5,02 & 9,30 & 8,12 & 7,37 & ns & $* *$ & $* *$ & $* *$ & $* *$ & $* *$ & $* *$ & $* *$ & $* *$ & $*$ \\
\hline 1990 & 4,38 & 4,74 & 2,59 & 3,52 & 3,73 & $\mathrm{~ns}$ & $* *$ & $* *$ & $* *$ & $* *$ & $* *$ & $* *$ & $* *$ & $* *$ & $\mathrm{~ns}$ \\
\hline 1991 & 6,78 & 7,69 & 7,08 & 7,97 & 7,61 & $*$ & ns & $* *$ & $*$ & $*$ & ns & $\mathrm{ns}$ & $* *$ & $*$ & $\mathrm{~ns}$ \\
\hline Média & 5,58 & 6,22 & 4,83 & 5,75 & 5,67 & ns & ns & ns & ns & ns & ns & $\mathrm{ns}$ & ns & ns & $\mathrm{ns}$ \\
\hline
\end{tabular}

${ }^{1}$ Sistema I = triticale/soja; Sistema II = triticale/soja e aveia preta ou aveia branca/soja; Sistema III = triticale/soja, e ervilhaca/milho; Sistema IV = triticale/soja, ervilhaca/milho e aveia preta ou aveia branca/soja; Sistema V = triticale/soja, triticale/soja, ervilhaca/milho e aveia preta ou aveia branca/soja.

ns = não significativo.

$*$ = significativo a $5 \%$ de probabilidade.

$* *$ = significativo a $1 \%$ de probabilidade.

dentre os sistemas estudados. SANTOS et al. (1996a, 1996b), estimando índices de produtividade cultural (resultado da divisão do rendimento de grãos de cada espécie pela energia consumida) para sistemas de rotação de culturas para cevada e trigo, durante dez anos, sob plantio direto, no Estado do Paraná, obtiveram diferenças significativas entre as médias. Os sistemas de rotação de culturas para cevada e para trigo foram mais eficientes energeticamente do que a monocultura cevada/soja ou trigo/soja.

Quando as culturas produzem rendimentos elevados, o efeito da rotação de culturas sobre a monocultura torna-se mais evidente; isso foi verdadeiro no primeiro período deste trabalho. Quanto mais elevado o rendimento de grãos, maior poderá ser a conversão energética das espécies.

$\mathrm{Na}$ análise da conversão energética (inverno + verão) do segundo período (1990 a 1991), observam-se diferenças significativas somente entre as médias anuais (tabela 2). Nesse período, o milho, quando produzido, rendeu menos (de $5.419 \mathrm{~kg} / \mathrm{ha}$ a $5.800 \mathrm{~kg} / \mathrm{ha}$ ) que nos anos anteriores. A cultura de soja igualmente teve produção baixa em 1990 (de $1.005 \mathrm{~kg} / \mathrm{ha}$ a $1.276 \mathrm{~kg} / \mathrm{ha})$ e alta em 1991 $(2.630 \mathrm{~kg} / \mathrm{ha}$ a $3.186 \mathrm{~kg} / \mathrm{ha})$; isso achatou os índices de conversão energética.

$\mathrm{Na}$ média conjunta do segundo período, para conversão energética, o sistema I (monocultura triticale/soja $=5,58$ ) não diferiu significativamente dos sistemas II $(6,22)$, III $(4,83)$, IV $(5,75)$ e V $(5,67)$ (tabela 2). Deve ser levado em conta que os sistemas III, IV e V tinham a cultura de milho como um de seus componentes e essa gramínea não foi colhida em 1990, devido à forte estiagem que ocorreu, principalmente na fase inicial de florescimento da cultura. Isso causou acentuado decréscimo nos valores da conversão desses sistemas e indica a importância e o potencial que tem a cultura de milho como conversora de energia. No ano seguinte, os sistemas IV e V foram superiores aos sistemas I e III. SANTOS et al. (1995), trabalhando com índices de eficiência energética, também não encontraram diferenças significativas entre os sistemas de rotação de culturas para trigo, num período de dez anos, sob sistema de preparo convencional de solo, no inverno, e sob plantio direto, no verão, em área relativamente próxima desse experimento.

No Canadá, ZENTNER et al. (1984), estudando 12 sistemas de rotação de culturas para trigo, durante 12 anos, verificaram diferenças significativas para conversão energética de um $(0,93)$ e em dois invernos sem trigo $(0,97)$, em comparação à monocultura desse cereal $(0,68)$. Os mesmos sistemas, avaliados 18 anos após, não apresentaram diferenças significativas entre as médias (ZENTNER $\boldsymbol{e t}$ al., 1989). Em ambos os casos, a cultura de verão não foi semeada. Na primeira fase desse estudo, evidenciou-se o efeito da rotação de culturas sobre a monocultura. 
Tabela 3 - Balanço energético e sua comparação em cinco sistemas de rotação de culturas para triticale, no ano (inverno + verão) e na média conjunta pelo teste $\mathrm{F}$, utilizando-se o método de contrastes.

\begin{tabular}{|c|c|c|c|c|c|c|c|c|c|c|c|c|c|c|c|}
\hline \multirow[b]{2}{*}{ Ano } & \multicolumn{5}{|c|}{ Sistema de rotação $^{1}$} & \multirow[b]{2}{*}{ I x II } & \multirow[b]{2}{*}{ I x III } & \multirow[b]{2}{*}{ I x IV } & \multirow[b]{2}{*}{ I x V } & \multirow[b]{2}{*}{ II x III } & \multirow[b]{2}{*}{ II x IV } & \multirow[b]{2}{*}{ II $x \mathrm{~V}$} & \multirow[b]{2}{*}{ III x IV } & \multirow[b]{2}{*}{ III $x$ V } & \multirow[b]{2}{*}{ IV $x \mathrm{~V}$} \\
\hline & I & II & III & IV & $\mathrm{V}$ & & & & & & & & & & \\
\hline 1987 & 16.152 & 10.765 & $\begin{array}{c}\text { Mcal/ha } \\
19.775\end{array}$ & 17.071 & 15.430 & ---- & $*$ & ns & ns & $\begin{array}{l}\text { ntrastes } \\
* *\end{array}$ & $\begin{array}{l}\text { entre sist } \\
* *\end{array}$ & $\begin{array}{l}\text { emas --- } \\
* *\end{array}$ & $*$ & $*$ & ns \\
\hline 1988 & 19.015 & 17.101 & 24.864 & 22.286 & 21.822 & $\mathrm{~ns}$ & $*$ & $\mathrm{~ns}$ & ns & $* *$ & $* *$ & $* *$ & $\mathrm{~ns}$ & ns & ns \\
\hline 1989 & 19.035 & 13.503 & 26.941 & 20.267 & 20.539 & $* *$ & $* *$ & $\mathrm{~ns}$ & ns & $* *$ & $* *$ & $* *$ & $* *$ & $* *$ & $\mathrm{~ns}$ \\
\hline Média & 18.067 & 13.790 & 23.860 & 19.875 & 19.264 & $*$ & $* *$ & ns & $\mathrm{ns}$ & $* *$ & $* *$ & $* *$ & $* *$ & $*$ & $\mathrm{~ns}$ \\
\hline 1990 & 14.191 & 15.705 & 6.932 & 10.781 & 11.586 & $\mathrm{~ns}$ & $* *$ & $* *$ & $* *$ & $* *$ & $* *$ & $* *$ & $* *$ & $* *$ & ns \\
\hline 1991 & 23.094 & 25.399 & 21.096 & 24.674 & 24.026 & $\mathrm{~ns}$ & ns & $\mathrm{ns}$ & $\mathrm{ns}$ & $* *$ & $\mathrm{~ns}$ & $\mathrm{~ns}$ & $* *$ & $* *$ & $\mathrm{~ns}$ \\
\hline Média & 18.643 & 20.552 & 14.014 & 17.728 & 17.806 & $\mathrm{~ns}$ & ns & ns & $\mathrm{ns}$ & ns & $\mathrm{ns}$ & ns & $\mathrm{ns}$ & ns & $\mathrm{ns}$ \\
\hline
\end{tabular}

${ }^{1}$ Sistema I = triticale/soja; Sistema II = triticale/soja e aveia preta ou aveia branca/soja; Sistema III = triticale/soja, e ervilhaca/milho; Sistema IV = triticale/soja, ervilhaca/milho e aveia preta ou aveia branca/soja; Sistema V = triticale/soja, triticale/soja, ervilhaca/milho e aveia preta ou aveia branca/soja. ${ }^{2}=$ quilograma/Mcal: Megaquicaloria (Kcal x 1.000).

ns = não significativo.

* = significativo a $5 \%$ de probabilidade.

** = significativo a $1 \%$ de probabilidade

É importante salientar que, no presente estudo, a conversão anual de energia de todos os sistemas avaliados foi superior à unidade, variando de 2,59 a 9,91 (tabela 2), significando que todos eles são conversores positivos de energia, ou seja, produziram 2,59 a 9,91 vezes mais energia do que a consumida (energia não renovável). Isso caracteriza um balanço energético positivo entre os sistemas estudados (QUESADA \& BEBER, 1990). Nesse caso, todos os sistemas estudados tornam a agricultura mais eficiente em termos de custo-benefício na produção de alimentos.

Na média do período 1987 a 1989, em termos de balanço energético (tabela 3 ), o sistema I (18.067 Mcal/ha) superou o sistema II (13.790Mcal/ha), foi inferior ao sistema III (23.860Mcal/ha) e não diferiu dos sistemas IV (19.875Mcal/ha) e V (19.264Mcal/ha). O sistema II foi superado pelos demais sistemas, enquanto o sistema III, na média geral do período, foi superior a todos. De modo geral, esses resultados repetiram-se, dentro de cada ano, nas análises estatísticas anuais. Conclui-se que o sistema III é a melhor opção, ou seja, é uma alternativa com resultado, em termos de energia, superior ao sistema tradicional de monocultura triticale/soja (sistema I) e aos demais sistemas alternativos, enquanto os sistemas IV e V podem ser indicados como alternativas ao sistema I, porém com a expectativa de se obter apenas dados semelhantes estatisticamente a este.

Nesse primeiro período de estudo, quando as culturas produziram bem, maior foi o aproveita- mento energético das espécies . Como a monocultura tendeu a produzir menos do que os sistemas com rotação de culturas, o seu aproveitamento energético foi menor.

A comparação dos balanços energéticos, no período 1990 a 1991, dentro de cada ano, mostrou muitas diferenças significativas entre os sistemas estudados em 1990, e poucas em 1991 (tabela 3). Essa discrepância de resultado entre os anos deve-se principalmente ao fato de que os sistemas III, IV e V, que tinham a cultura de milho como um de seus componentes, em 1990, tiveram acentuado decréscimo nos seus valores do balanço energético, porque essa gramínea não foi colhida, devido à forte estiagem que ocorreu na fase de florescimento da cultura. Em 1990, o sistema I não diferiu do sistema II e foi superior aos sistemas III, IV e V; no entanto, no ano seguinte, o sistema I não diferiu dos demais sistemas na totalidade dos casos.

A comparação das médias gerais dos sistemas, no período 1990 a 1991, não mostrou nenhum contraste significativo, indicando que os sistemas estudados não diferiram entre si em energia líquida. É importante salientar que, no presente estudo, nos dois períodos, todos os sistemas avaliados apresentaram balanço energético positivo, significando que todos eles produziram mais energia do que consumiram.

Nos estudos conduzidos no Canadá, com 12 sistemas de rotação de culturas para trigo, durante 12 anos, ZENTNER et al. (1984) não observaram 
diferenças significativas para o balanço energético entre um (10.868 Mcal/ha) ou dois invernos sem trigo $(10.590 \mathrm{Mcal} / \mathrm{ha})$ e a monocultura dessa gramínea (11.896 Mcal/ha ). Da mesma forma, os mesmos sistemas, avaliados 18 anos após, não apresentaram diferenças significativas nas médias do balanço energético entre a monocultura desse cereal e os sistemas de rotação de culturas para trigo (ZENTNER et al., 1989). Deve ser levado em consideração que, em ambos os casos, não foi semeada a cultura de verão.

As tecnologias agrícolas utilizadas nos sistemas estudados foram mais eficientes em termos de conversão energética e de balanço energético. Além disso, no primeiro período de estudo, o sistema III (triticale/soja e ervilhaca/milho) foi o mais eficiente dentre os sistemas avaliados. Nesse caso, somente a diversificação de espécies tornou esse sistema mais eficiente sem aumentar o consumo de energia não renovável (exemplo: combustíveis, fertilizantes, fungicidas, herbicidas e inseticidas).

\section{CONCLUSÕES}

1) Quando as condições climáticas transcorreram normalmente à produção agrícola (1987 a 1989), o sistema III foi o mais eficiente energeticamente dentre os demais sistemas estudados.

2) Quando as condições climáticas ocorreram adversamente à produção agrícola (1990 e 1991), não houve diferenças significativas entre as médias para conversão energética e para balanço energético.

3) Todos os sistemas avaliados foram conversores positivos de energia.

\section{REFERÊNCIAS BIBLIOGRÁFICAS}

BERARDI, G.M. Organic and conventional wheat production: examination of energy and economics. Agro-Ecosystems, Amsterdam, v. 4, n. 3, p. 367-376, 1978.

BOHRA, C.P., VARSHNEY, A.C, NARANG, S. Energy and cost audit of bullock and power tiller farming system in soybean and wheat crop production. Journal of Scientific and Industrial Research, Bhopal, IND v. 49, n. 12, p. 583-588, 1990.

BRASIL. Ministério da Agricultura. Departamento Nacional de Pesquisa Agropecuária. Divisão de Pesquisa Pedológica Levantamento de reconhecimento dos solos do estado do Rio Grande do Sul. Recife, 1973, 421p., Boletim Técnico, 60

DERPSCH, R., CALEGARI, A. Plantas para adubação verde de inverno. Londrina: IAPAR, 1992, 80 p. IAPAR. Circular, 73.

DERPSCH, R., SIDIRAS, N., HEINZMANN, F.X. Manejo do solo com coberturas verdes de inverno. Pesquisa Agropecuária Brasileira, Brasília, v. 20, n. 7, p. 761-773, jul. 1985.
EMBRAPA. Centro Nacional de Pesquisa de Suínos e Aves (Concórdia, SC). Tabela de composição química e valores energéticos de alimentos para suínos e aves. 3.ed. Concórdia: Embrapa-CNPSA, 1991. 97 p. Documentos, 19.

HEICHEL, G. H. Assessing the fossil energy costs of propagating agricultural crops. In: PIMENTEL, D. (Ed.). Handbook of energy utilization in agriculture. Boca Raton: CRC, 1980, p. 27-33.

IGUE, K. Energia e agricultura. In: IAPAR. Manual agropecuário para o Paraná. Londrina: IAPAR, 1980, v. 3, cap. 9 , p. $217-228$

MARCHIORO, N. de P.X. Balanço ecoenergético: uma metodologia de análise de sistemas agrícolas. In: TREINAMENTO EM ANÁLISE ECOENERGÉTICA DE SISTEMAS AGRÍCOLAS, 1., 1985, Curitiba. Anais... Curitiba: IAPAR, 1985.75 p. p. 24-40.

MELLO, R. de. Análise energética de agroecosssistemas: o caso de Santa Catarina. Florianópolis, 1986, 139 p. Dissertação (Mestrado em Engenharia) - Curso de Pósgraduação em Erngenharia de Produção, UFSC, 1986.

PIMENTEL, D. Handbook of energy utilization in agriculture. Boca Raton: CRC, 1980, 475 p.

QUESADA, G.M., BEBER, J.A.C. Energia e mão-de-obra. Ciência Hoje, Rio de Janeiro, v. 11, n. 62, p. 21-26, 1990.

QUESADA, G.M., BEBER, J.A.C., SOUZA, S.P. de. Balanços energéticos agropecuários. uma proposta metodológica para o Rio Grande do Sul. Ciência e Cultura, São Paulo, v. 39, n. 1 , p. 20-28, 1987.

SANTOS, H.P. dos, IGNACZAK, J.C., LHAMBY, J.C.B. Produtividade cultural de sistemas de rotação de culturas para trigo, num período de dez anos, em Passo Fundo, RS. Pesquisa Agropecuária Brasileira, Brasília, v. 30, n. 12, p. 1397-1402, dez. 1995.

SANTOS, H.P. dos, IGNACZAK J.C. SANDINI, I. Produtividade cultural de sistemas de rotação de culturas para cevada, sob plantio direto. Pesquisa Agropecuária Brasileira, Brasília, v. 31, n. 10, p. 721-727, out. 1996 a.

SANTOS, H.P. dos, IGNACZAK, J.C., WOBETO, C. Produtividade cultural de sistemas de rotação de culturas com o trigo, sob plantio direto. Pesquisa Agropecuária Brasileira, Brasília, v. 31, n. 4, p. 277-282, abri. 1996b.

STEEL, R.G.D., TORRIE, J.H. Principles and procedures of statistics: a biometrical approach. 2. ed. New York: McGrawHill, 1980, 633 p.

WHITE, D.J. Energy in agricultural systems. The Agricultural Engineer, Bedford, ENG, v. 30, n. 3, p. 52-58, 1975.

WILSON, P.N., BRIGSTOCKE, T.D.A. Energy usage in Bristish agriculture - a review of future prospects. Agricultural Systems, Essex, UKv.5, p. 51-70, 1980.

ZENTNER, R.P., CAMPBELL, D.W., CAMPBELL, C.A., $\boldsymbol{e} \boldsymbol{t}$ al. Energy consideration of crop rotation in Southwestern Saskatchewan. Canadian Agricultural Engineering, Ottawa, v. 26, n. 1 , p. $25-29,1984$

ZENTNER, R.P., STUMBORG, M.A., CAMPBELL, C.A. Effect of crop rotations and fertilization on energy balance in typical production systems on the Canadian prairies. Agriculture, Ecosystems and Environment, Amsterdam, v. 25, n. 2-3, p. 217-232, 1989. 